\title{
Erythrocyte and Reticulocyte Indices on the LH 750 as Potential Markers of Functional Iron Deficiency
}

\author{
Eloísa Urrechaga, ${ }^{1}$ Luís Borque, ${ }^{2}$ and Jesús F. Escanero ${ }^{2}$ \\ ${ }^{1}$ Hematology Laboratory, Hospital Galdakao-Usansolo, Galdakao, 48960 Vizcaya, Spain \\ ${ }^{2}$ Department of Pharmacology and Physiology, Faculty of Medicine, University of Zaragoza, Zaragoza, Spain
}

Correspondence should be addressed to Eloísa Urrechaga, eloisa.urrechagaigartua@osakidetza.net

Received 20 December 2009; Accepted 31 March 2010

Academic Editor: Fernando Ferreira Costa

Copyright ( 2010 Eloísa Urrechaga et al. This is an open access article distributed under the Creative Commons Attribution License, which permits unrestricted use, distribution, and reproduction in any medium, provided the original work is properly cited.

\begin{abstract}
Reticulocyte hemoglobin content (CHr) and percentage of hypochromic cells (\%Hypo) are restricted to the Siemens analysers. The aims of the study were to investigate the correlation of Red cells size factor (RSf) and low Hemoglobin density (LHD\%), reported by Beckman-Coulter analysers, with $\mathrm{CHr}$ and \%Hypo in the assessment of iron status in the presence of inflammation. 381 samples were run on both LH 750 (Beckman-Coulter) and Advia 2120 (Siemens) analysers. Correlation between parameters were calculated and the diagnostic performance of the new parameters was assessed. Results. Correlation between $\mathrm{RSf}$ and $\mathrm{CHr}$, $r=0.85$. ROC curve analysis for RSf in the diagnosis of iron restricted erythropoiesis defined as CHr < 28 pg: AUC 0.983; Cutoff 91.1\%; Sensitivity 98.8\%; Specificity 89.6\% Correlation between LHD\% and \%Hypo, $r=0.869$. ROC curve analysis for LHD $\%$ in the diagnosis of iron deficiency defined by \%Hypo > 5\%: AUC 0.954; Cut off 6.0\%; Sensitivity $96.6 \%$; Specificity $83.2 \%$ Conclusions. RSf and LHD\% could be reliable parameters for the study of iron metabolism status.
\end{abstract}

\section{Introduction}

In iron deficiency anemia (IDA) iron supply depends on the quantity of iron storage in the body, while in functional iron deficiency (iron restricted erythropoiesis) supply depends on the rate of mobilization of iron from the stores. Functional iron deficiency is defined as an imbalance between the iron needs for erythropoiesis and the iron supply, with the latter not maintained at sufficient rate for adequate hemoglobinization of reticulocytes and mature erythrocytes [1].

The diagnosis of iron deficiency or functional iron deficiency is particularly challenging in patients with acute or chronic inflammatory conditions because most of the biochemical markers for iron metabolism are affected by acute phase reaction. This is the case of the anemia of chronic disease $(\mathrm{ACD})$ and the anemia associated to chronic renal failure (CKD).

Serum ferritin, an indicator of iron storage but not of iron supply, is a positive acute phase reactant, while transferrin is a negative acute phase reactant, rendering the calculation of transferrin saturation unreliable in this case [2-4].

For these reasons, an iron deficient erythropoiesis may occur despite that normal serum ferritin and transferrin saturation values and interest have been generated in the use of erythrocyte and reticulocyte parameters, available on the modern analysers based on flow cytometry technology.

Direct consequence of an imbalance between the erythroid marrow iron requirements and the actual supply is a reduction of red cell hemoglobin content, which causes hypochromic mature red cells and reticulocytes.

Reticulocyte hemoglobin content $(\mathrm{CHr})$ and the percentage of hypochromic red blood cells (\%Hypo) reflect iron availability and are reliable markers of functional iron deficiency $[5,6]$.

The measurement of $\mathrm{CHr}$ is a direct assessment of the incorporation of iron into erythrocyte hemoglobin $(\mathrm{Hb})$ and thus an estimate of the recent functional availability of iron into the erythron; due to the life span of the reticulocytes, $\mathrm{CHr}$ is a sensitive indicator of iron deficient erythropoiesis [7-9]. 
The measurement of \%Hypo (defined as the percentage of red blood cells with $\mathrm{Hb}$ concentration less than $280 \mathrm{~g} / \mathrm{L}$ ) is a sensitive method for quantifying the hemoglobinization of mature red cells. Because of the long circulating life span of mature erythrocytes \%Hypo values are related to iron status in the last 2-3 months, and have been recognised as an indicator of iron deficiency $[10,11]$.

$\mathrm{CHr}$ and \%Hypo have been used as a diagnostic tool, together with biochemical markers, to distinguish IDA from ACD, and are incorporated to National Kidney Foundation (NKF-K/DOQI) guidelines for the monitoring of recombinant human erythropoietin $\mathrm{rHuEPO}$ therapy [12-14].

To date, the measurement of $\mathrm{CHr}$ and \%Hypo has been restricted to the analysers of a single manufacturer, Siemens (Siemens Medical Solutions Diagnostics, Tarrytown N.Y, USA). A second manufacturer has produced a comparable index, the so-called reticulocyte hemoglobin equivalent (Ret $\mathrm{He}$ ) generated by the Sysmex XE 2100 analyser (Sysmex Corporation, Kobe, Japan).

Measurements of Ret He provides useful information in diagnosing anemia, iron restricted erythropoiesis, and functional iron deficiency and response to iron therapy during r-HuEPO. Twenty nine pg is the cutoff value that defines deficient erythropoiesis $[15,16]$. Ret He correlates with $\mathrm{CHr}$ with the same clinical meaning [17]. The new Symex XE 5000 analyser reports the percentages of hypochromic red cells, but few data are already published about this parameter [18].

Beckman Coulter (Beckman Coulter Inc., Miami, Fl, USA) has recently introduced on the LH series analysers two new parameters

Red blood cell Size Factor (RSf) joins together the volume of mature red cells (MCV) and the volume of reticulocytes (MRV), both related to erythropoietic activity and hemoglobinization

$$
\mathrm{RSf}=\sqrt{\mathrm{MCV}^{*} \mathrm{MRV}} .
$$

Compared to the mature erythrocyte population, reticulocytes have a mean volume 15-20 fL greater, they stay in the blood stream for 1-1.5 days, so the measurement of reticulocyte number and cellular characteristics, such as volume, provides real-time data regarding certain aspects of erythropoiesis that can influence the dimensions of red cells, such as iron availability. The examination of both precursors and mature cells provides an opportunity to detect and monitor acute and chronic changes in cellular hemoglobin status, related to cell volume [19].

Low hemoglobin density (LHD\%) derives from the traditional mean cell hemoglobin concentration (MCHC), using the mathematical sigmoid transformation

$$
\text { LHD } \%=100 * \sqrt{1-\left(\frac{1}{\left(1+e^{1.8(30-\mathrm{MCHC})}\right)}\right)} .
$$

$\mathrm{MCHC}$ is an all-inclusive measure of both the availability of iron over the preceding 90-120 days, and of the proper introduction of iron into intracellular hemoglobin.

In the same way, $\mathrm{LHD} \%$ is related to iron availability and the hemoglobinization of the mature red cells.
In this equation defining $\mathrm{LHD} \%$, in addition to the standard sigmoid function, a square root is applied to further enhance numerical resolution in the region corresponding to the lower end of \%Hypo, to improve the differentiation between the normal and the abnormal among the blood samples having relatively low values of LHD\% [20].

The aims of this study were

(i) to establish the reference range of RSf and LHD\% and their values in different types of anemia;

(ii) to evaluate these recently introduced reticulocyte and erythrocyte parameters provided by the $\mathrm{LH}$ series analysers in terms of correlation with $\mathrm{CHr}$ and \%Hypo as well as their diagnostic efficiency assuming $\mathrm{CHr}<28.0 \mathrm{pg}$ or \%Hypo $>5 \%$ to detect iron restricted erythropoiesis and iron deficiency.

\section{Materials and Methods}

2.1. Criteria for Selecting the Groups of Patients. Samples from 120 healthy individuals, 72 iron deficiency anemia (IDA), 60 IDA with acute phase response (IDA APR), 71 chronic kidney disease (CKD), and 58 anemia of chronic disease $(A C D)$ were randomly extracted from the routine workload and run sequentially on both LH 750 (Beckman Coulter Inc. Miami, Fl, USA) and Advia 2120 (Siemens Medical Solutions Diagnostics, Tarrytown N.Y., USA), analysers within 6 hours of collection.

Healthy group: 54 male and 66 female adult subjects, with no clinical symptoms of disease and with results of the complete blood count and biochemical iron metabolism markers within reference ranges.

A group of 132 IDA patients fulfilled traditional diagnostic criteria for iron deficiency anemia diagnosis, serum iron < $7.5 \mu \mathrm{mol} / \mathrm{L}$, transferrin saturation $<20 \%$, ferritin $<50 \mu \mathrm{g} / \mathrm{L}$, and $\mathrm{Hb}<110 \mathrm{~g} / \mathrm{L}$, were included before iron treatment.

This group was divided into a nonacute phase response group $(n=72, \mathrm{CRP}<5 \mathrm{mg} / \mathrm{L})$ and acute phase response group $(n=60, \mathrm{CRP}>5 \mathrm{mg} / \mathrm{L})$. Acute phase response included inflammation or infectious conditions, in addition to ferropenic status.

CKD patients were managed according to the recommendations of the NKF-K/DOQI guidelines [21]. All patients were treated with a variety of erythropoietin doses, the majority of them were treated with a maintenance dose of intravenous iron weekly, in order to maintain $\mathrm{Hb}$ at the recommended level $110-120 \mathrm{~g} / \mathrm{L}$.

ACD group included patients with a variety of diseases: chronic infections (tuberculosis); neoplasic disorders (Hodgkin's disease, breast carcinoma); noninfectious inflammatory diseases (rheumatoid arthritis, systemic lupus erythematosus).

ACD patients received treatment to maintain normal erythropoiesis and presented the traditional diagnostic criteria for "Functional iron-deficiency" diagnosis Transferrin saturation $<20 \%$, $\mathrm{Hb}<110 \mathrm{~g} / \mathrm{L}$, and serum ferritin values normal or over the reference range.

Table 1 shows the hematological and biochemical data of the different groups. 
TABLE 1: Hematological and biochemical data of the healthy group $(n=120)$, iron deficiency anemia (IDA, $n=72)$, iron deficiency anemia and acute phase response (IDA APR, $n=60$ ), anemia of chronic disease (ACD, $n=58$ ), and chronic kidney disease (CKD, $n=71)$ patients.

\begin{tabular}{|c|c|c|c|c|c|c|c|c|c|}
\hline & $\mathrm{RBC} 10^{12} / \mathrm{L}$ & $\mathrm{Hb} \mathrm{g} / \mathrm{L}$ & MCV fL & $\mathrm{MCH}$ pg & $\mathrm{MCHC} g / \mathrm{L}$ & Iron $\mu \mathrm{mol} / \mathrm{L}$ & Transf g/L & Ferritin $\mu \mathrm{g} / \mathrm{L}$ & Sat $\%$ \\
\hline Healthy mean & 4.9 & 154 & 91.1 & 31.3 & 343 & 16.5 & 2.53 & 75 & 31 \\
\hline$(\mathrm{SD})$ & $(0.27)$ & (6.4) & $(2.55)$ & $(1.53)$ & $(5.2)$ & $1(0.62)$ & $(0.2)$ & $(2.8)$ & (1.9) \\
\hline IDA mean & 4.6 & 95 & 70 & 22.5 & 320 & 4.8 & 3.31 & 14 & 6 \\
\hline$(\mathrm{SD})$ & $(0.61)$ & $(14.2)$ & $(10.3)$ & $(4.23)$ & $(17.3)$ & $(2.15)$ & $(0.53)$ & (9) & (3.6) \\
\hline IDA APR mean & 4.4 & 96 & 75.8 & 21.5 & 327 & 5.1 & 2.78 & 37 & 9 \\
\hline$(\mathrm{SD})$ & $(0.43)$ & $(12.1)$ & (3.7) & (1.3) & $(9.2)$ & $(3.5)$ & $(0.28)$ & (25) & (5.6) \\
\hline ACD mean & 3.5 & 101 & 93.2 & 31.9 & 343 & 10.0 & 2.68 & 522 & 15 \\
\hline$(\mathrm{SD})$ & $(0.48)$ & $(11)$ & $(6.0)$ & $(2.23)$ & (10) & $(6.8)$ & $(0.66)$ & $(704)$ & (5) \\
\hline CKD mean & 3.5 & 112 & 95.6 & 31.1 & 325 & 9.8 & 1.87 & 335 & 21 \\
\hline$(\mathrm{SD})$ & $(0.45)$ & $(8.5)$ & $(6.67)$ & $(2.23)$ & $(8)$ & $(4.47)$ & $(0.43)$ & (204) & (10) \\
\hline
\end{tabular}

RBC, red blood cells; $\mathrm{Hb}$, hemoglobin; MCV, mean cell volume; $\mathrm{MCH}$, mean cell hemoglobin; MCHC, mean cell hemoglobin concentration; Transf, transferrin; Sat, \% transferrin saturation.

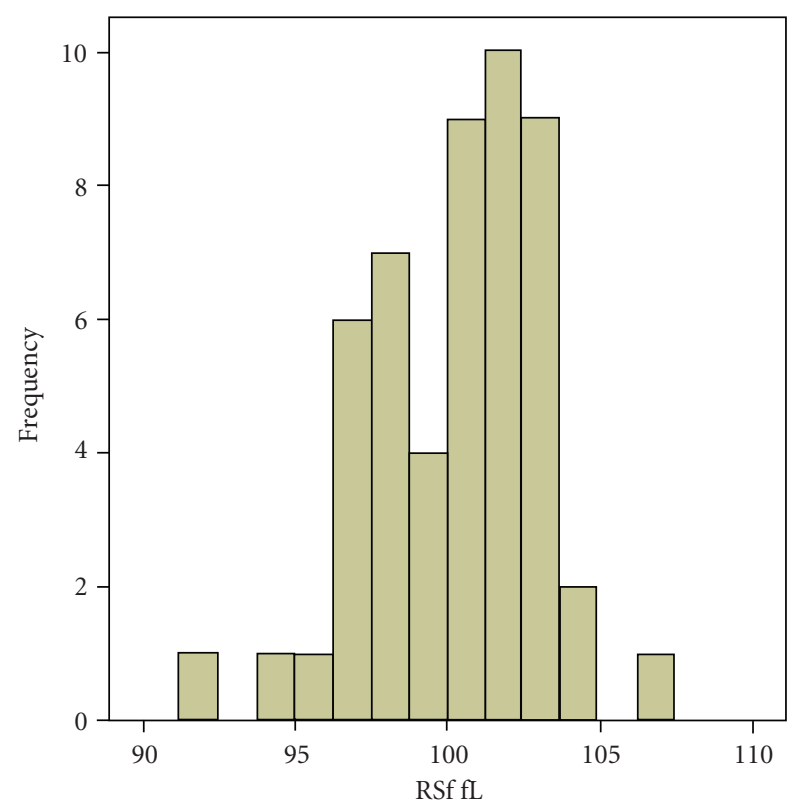

FIGURE 1: Red blood cell size factor (RSf) values in a population of 120 healthy adult subjects. The values showed Gaussian distribution (Kolmogorov-Smirnoff test, $P=.279$ ).

2.2. Statistical Evaluation of Analytical Results. Statistical software package SPSS (SPSS; Chicago, IL, USA) version 17.0 for Windows was applied for statistical analysis of the results.

Reference ranges were calculated from the results obtained in the group of healthy subjects (95 central percentiles of the distribution). Kolmogorov-Smirnoff test was applied to verify the Gaussian distribution of RSf and LHD\% values.

When the parameters under study presented a Gaussian distribution correlation, coefficients were calculated by Pearson method; independent samples $t$-test was performed in order to detect statistical deviations between the groups of patients; $P$ values less than .05 were considered to be statistically significant.

When the parameters under study presented a nonGaussian distribution correlation, coefficients were calcu-

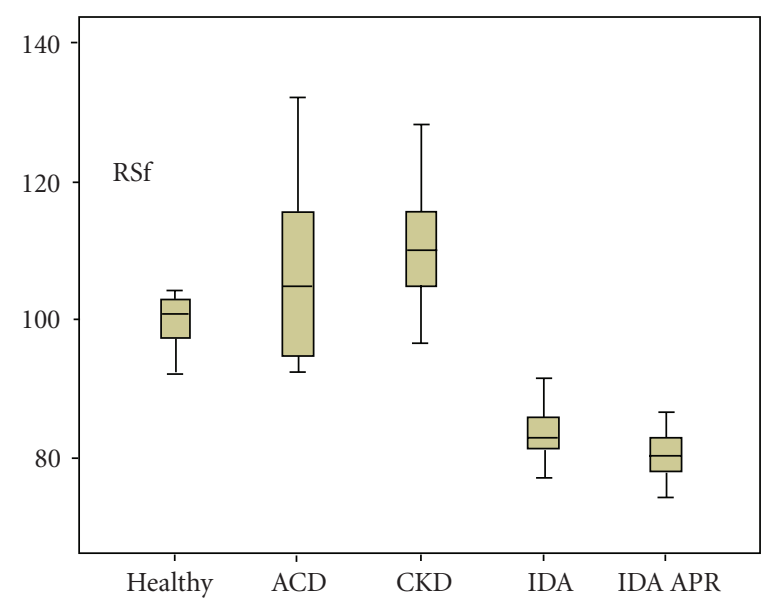

FIGURE 2: Comparison of red blood cell size factor (RSf) in the groups of anemic patients, anemia of chronic disease (ACD), chronic kidney disease (CKD), iron deficiency anemia (IDA) iron deficiency anemia and acute phase response (IDA APR), and in the healthy group.

lated by Spearman method; independent samples MannWhitney $U$-test was performed; $P$ values less than .05 were considered to be statistically significant.

Receiver operating characteristic (ROC) curve analysis was utilized to illustrate the diagnostic performance of RSf and $\mathrm{LHD} \%$ in the detection of iron deficiency status, defined by a $\mathrm{CHr}$ value $28 \mathrm{pg}$.

Cutoff values were established based on the optimal combination of sensitivity and specificity.

\section{Results}

The values of RSf were normally distributed $(P=.279)$ (Figure 1). Reference range was 91.1-106.9 fL.

Figure 2 and Table 2 exhibit CHr and RSf mean values and standard deviation (SD) in the variety of anemia and healthy subjects included in the study.

The horizontal line in the center of the box shows the median value, the upper and lower limits of the box show the 


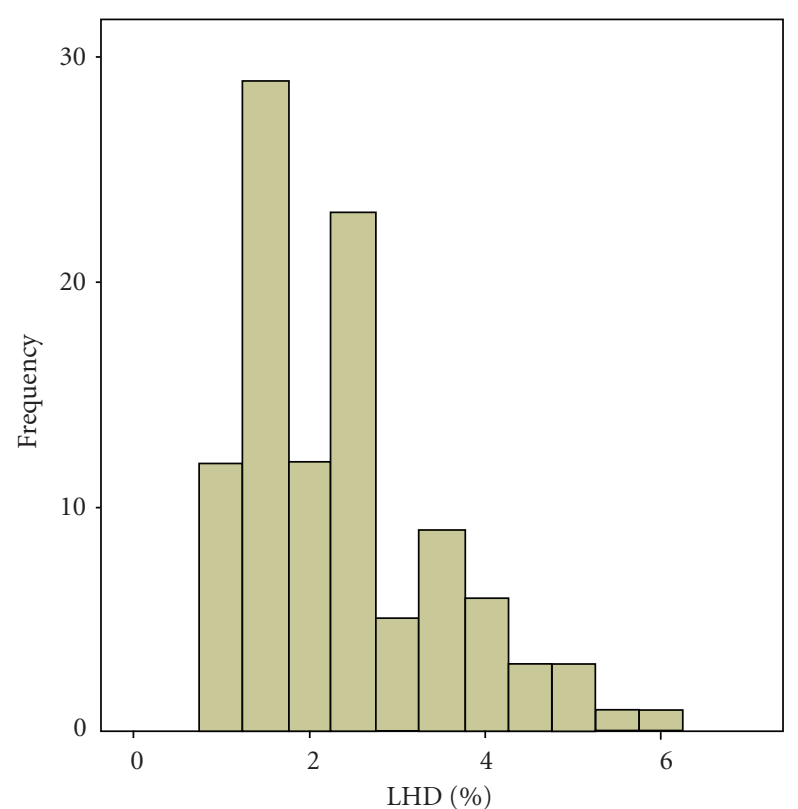

FIGURE 3: low hemoglobin density (LHD\%) values in a population of 120 healthy adult subjects. The values showed a non-Gaussian distribution (Kolmogorov-Smirnoff test, $P=.034$ ).

TABLE 2: Reticulocyte hemoglobin content $(\mathrm{CHr})$ and red blood cell size factor (RSf) values, mean and standard deviation (SD), in the group of patients.

\begin{tabular}{lcc}
\hline & CHr pgmean (SD) & RSf fLmean (SD) \\
\hline Healthy & $33.2(1.6)$ & $100.9(5.3)$ \\
IDA & $24.5(4.4)$ & $88.1(7.8)$ \\
IDA APR & $25.6(2.5)$ & $84.7(4.1)$ \\
ACD & $30.8(5.1)$ & $105.5(10.9)$ \\
CKD & $31.6(3.5)$ & $110.6(8.7)$ \\
\hline
\end{tabular}

interquartile range, and the whiskers show the minimum and maximum values for each group.

The horizontal line in the center of the box shows the median value, the upper and lower limits of the box show the interquartile range, and the whiskers show the minimum and maximum values for each group.

Correlation between $\mathrm{CHr}$ and RSf values, Pearson regression coefficient $0.85, P<.001$.

Independent samples $t$-test was performed in order to detect statistical deviations between the groups of patients.

Significant differences in RSf mean values $(P<.001)$ were detected when groups with iron restricted erythropoiesis (IDA, mean $88.1 \mathrm{fL}$ and IDA with APR, mean $84.7 \mathrm{fL}$ ) were compared with patients undergoing therapy (ACD, mean $108.9 \mathrm{fL}$ and CKD, $110.6 \mathrm{fL})$ and the healthy subjects (mean $100.9 \mathrm{fL}$ ).

No statistic difference was found between IDA group and IDA patients with acute phase response $(P=.481)$.

IDA and IDA with APR groups presented iron restricted erythropoiesis as is stated by $\mathrm{CHr}$ values $(24.5 \mathrm{pg}$ and $25.6 \mathrm{pg}$,

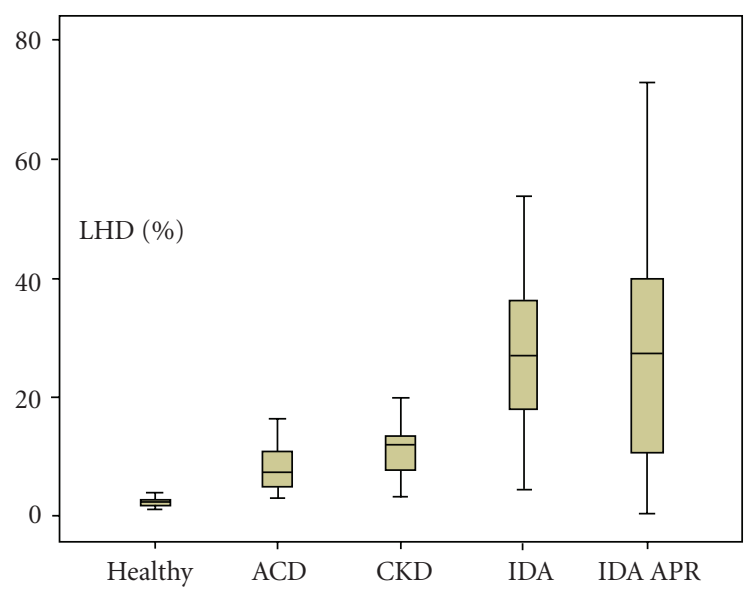

Figure 4: Comparison of low hemoglobin density (LHD\%) in the groups of anemic patients, anemia of chronic disease (ACD), chronic kidney disease (CKD), iron deficiency anemia (IDA) iron deficiency anemia and acute phase response (IDA APR), and in the healthy group.

TABle 3: Percentage of hypochromic red cells (\% Hypo) values, mean and standard deviation (SD) and low hemoglobin density (LHD\%) values, median (5th-95th interquartiles, IQ) in the group of patients.

\begin{tabular}{lcc}
\hline & \% Hypo Mean (SD) & LHD\% Median (IQ) \\
\hline Healthy & $0.13(0.15)$ & $2.1(0.9-4.1)$ \\
IDA & $17.2(17.4)$ & $29.6(7.5-76)$ \\
IDA APR & $16.8(15.5)$ & $27.3(8.3-71.2)$ \\
ACD & $4.1(4.4)$ & $7.3(5.1-30)$ \\
CKD & $5.1(6.7)$ & $9.6(5.6-27)$ \\
\hline
\end{tabular}

resp.) ACD and CKD patients receiving therapy maintained $\mathrm{CHr}$ levels higher than the cutoff value of $28 \mathrm{pg}$.

ROC curve analysis for RSf in the diagnosis of restricted erythropoiesis, defined by $\mathrm{CHr}<28 \mathrm{pg}$, results were AUC 0.983 , Cutoff $91.1 \mathrm{fL}$, sensitivity $98.8 \%$, specificity $89.6 \%$.

The values of LHD\% were not normally distributed ( $P=$ .034) (Figure 3). Reference range was $0 \%-4.4 \%$.

Figure 4 and Table 3 exhibit \%Hypo values, mean and standard deviation (SD) and LHD\% values, median and 5th95th interquartiles, in the variety of anemias and healthy subjects included in the study.

The horizontal line in the center of the box shows the median value, the upper and lower limits of the box show the interquartile range, and the whiskers show the minimum and maximum values for each group.

Correlation between \%Hypo and LHD\% values, $r=$ 0.869 (Spearman method) $(P<.001) . y=1.338 x+4.40$.

Independent samples $U$-test was performed in order to detect statistical deviations between the groups of patients.

Significant differences in LHD\% values $(P<.001)$ were detected when groups with iron deficiency (IDA, mean $29.6 \%$ and IDA with APR, mean 27.3\%) were compared with patients undergoing therapy $(\mathrm{ACD}$, mean $7.3 \%$; $\mathrm{CKD}$, mean 9.6\%) and the healthy subjects (median $2.1 \%$ ). 


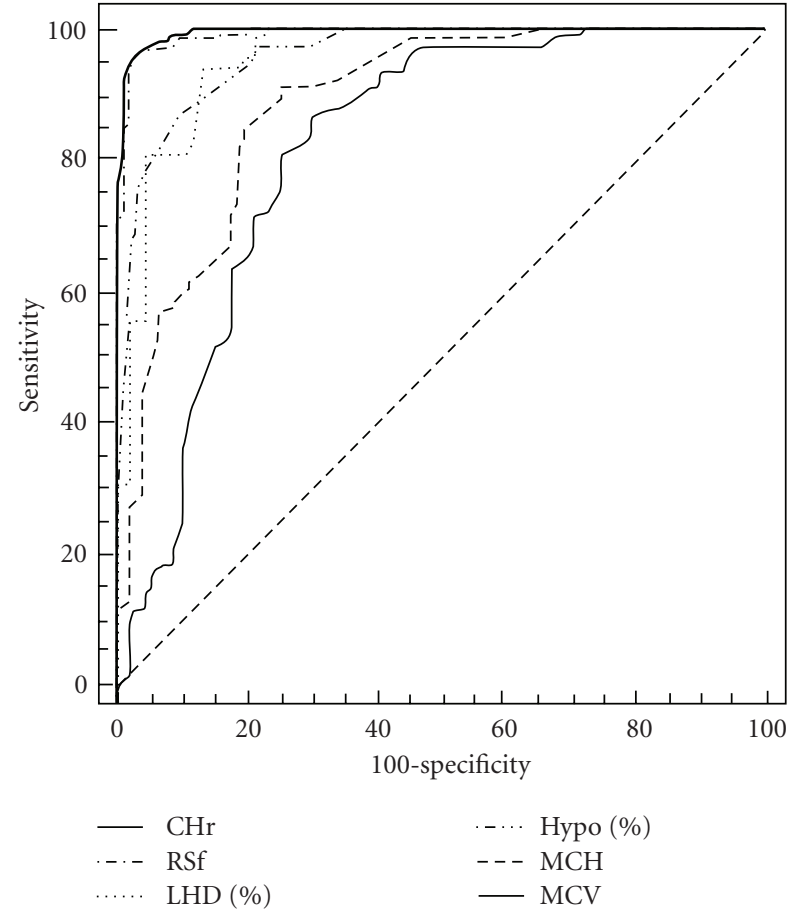

FIGURE 5: Receiver operating characteristic (ROC) curves for red blood cell size factor (RSf), low hemoglobin density (LHD\%), reticulocyte hemoglobin content $(\mathrm{CHr})$, percentage of hypochromic red cells (\% Hypo), mean cell hemoglobin $(\mathrm{MCH})$ and mean cell volume $(\mathrm{MCV})$ in the diagnosis of iron deficiency, defined by $\mathrm{CHr}$ $<28 \mathrm{pg}$.

No statistic difference was found between IDA group and IDA patients with acute phase response $(P=.578)$.

Receiver operating characteristic (ROC) curve analysis for $\mathrm{LHD} \%$ in the diagnosis of iron deficiency, defined by $\%$ Hypo > 5\% AUC 0.954, cutoff 6.0\%, sensitivity 96.6\%, specificity $83.3 \%$.

Discriminant efficiency of biochemical parameters and classical erythrocyte indices:

mean cell hemoglobin $(\mathrm{MCH})$, AUC 0.89; mean cell volume, (MCV), AUC 0.822; serum ferritin, AUC 0.722; serum iron, AUC 0.683 (Figure 5).

\section{Discussion}

Uncomplicated iron deficiency is not difficult to diagnose by means of the traditional laboratory tests. Biochemical markers are reliable parameters to diagnose iron deficiency in an uncomplicated clinical setting.

Serum ferritin, as a potent positive acute phase reactant is often increased in ACD and CKD patients [22]. Transferrin saturation is less than $15 \%$ to $20 \%$ in iron deficiency, but expression of serum transferrin is reported to be downregulated by inflammatory cytokines, so may not reliably reflect iron deficiency when anemia is complicated by inflammation [23].

Efforts have been made to evaluate some readily available and relatively inexpensive laboratory parameters as indirect markers of iron restricted erythropoiesis and iron availability in a clinical context influenced by inflammation and acute phase reaction.

The best combination of hematological indices for iron deficiency is an increased percentage of hypochromic erythrocytes and a reduced hemoglobin content of reticulocytes [24].

Measurements of reticulocyte hemoglobin content $(\mathrm{CHr})$ have been shown to provide useful information in diagnosing functional iron deficiency during $\mathrm{r}$-HuEPO therapy $[25,26]$ and response to iron therapy $[27,28]$.

Recent studies confirm the clinical reliability of mature erythrocyte parameters, such as hypochromic red cells (Hypo $\%$ ), as markers of iron deficiency in hemodialysis patients $[29,30]$.

Beckman Coulter (Beckman Coulter Inc. Miami, Fl, USA) applies the Volume Conductivity Scatter technology to this field and new parameters are now available on the LH series analysers.

The main purpose of the present study was to determine the diagnostic performance of RSf and LHD\% and to assess whether the new parameters correlate with the existing diagnostic tests $\mathrm{CHr}$ and \%Hypo for restricted erythropoiesis and functional iron deficiency diagnosis.

This study shows a reasonable level of agreement between $\mathrm{RSf}$ and $\mathrm{CHr}$.

Despite the fact that $\mathrm{CHr}$ is a measurement of the reticulocyte hemoglobin content and RSf is a measurement of both mature red cells and reticulocyte size, a high concordance between these parameters has been observed and values of both parameters showed the same trend in the different groups.

In patients with iron restricted erythropoiesis, (IDA and IDA APR) RSf values were lower than the reference range $91.1 \mathrm{fL}$.

ACD and CKD patients were receiving therapy, they suffered a mild anemia but maintained bone marrow activity, as the $\mathrm{CHr}$ values state (30.8 and $31.6 \mathrm{pg}$, resp.). RSf values in these patients were in the reference range of RSf, but the macrocytosis associated to CKD and hemodialysis treatment causes that $15 \%$ of the patients in this group had RSf values slightly over the reference range, $106.6 \mathrm{fL}$.

Diagnostic sensitivity, specificity, and efficiency of RSf were good when compared to a $28 \mathrm{pg}$ value of $\mathrm{CHr}$. In particular, a cutoff value of $91.1 \mathrm{fL}$ for RSf showed the best diagnostic efficiency, with a sensitivity of $98.8 \%$ and a specificity of $89.6 \%$.

This study shows a reasonable agreement between \% Hypo and LHD\% and their values showed the same trend in the different groups.

$\%$ Hypo values in patients undergoing therapy were near the threshold of iron deficient erythropoiesis 5\% (ACD 4.1\%, CKD 5.1\%).

The LHD\% values obtained in these groups of patients were statistically lower (ACD 7.3\%, CKD 9.6\%) than the iron deficient ones $(29.6 \%, 27.3 \%)$ but, as all of them had a mild anemia, LHD\% values in these patients were above the reference range $4.4 \%$.

The optimal cutoff point for LHD\% was $6.0 \%$, which provided sensitivity $96.6 \%$, specificity $83.2 \%$, and area under 
curve 0.954 , for iron deficiency detection, defined by \%Нypo $>5 \%$.

In this study, it has been stated that RSf and LHD\% could be reliable parameters for the study of iron status and the amount of the supply available for erythropoiesis.

Iron metabolism is a dynamic process which cannot be defined by one test parameter only. The analysis of these new parameters can be performed simultaneously in the course of routine blood counts, with no incremental costs and no additional needs of more blood sampling. In conjunction with standard blood cell counts and iron, parameters could enable the diagnosis to be made rapidly and accurately.

The new parameters derived from Beckman-Coulter technology seem to be an acceptable alternative to $\mathrm{CHr}$ and \%Hypo in the routine practice, with the same clinical meaning, but more prospective and longitudinal studies are needed in order to verify the results obtained, to determine their reliability for clinical purposes or whether the additional information provided could be used in managing the iron requirements of patients.

\section{References}

[1] I. Cavill and I. C. Macdougall, "Functional iron deficiency," Blood, vol. 82, no. 4, p. 137, 1993.

[2] S. Fishbane, L. J. Imbriano, E. A. Kowalski, and J. K. Maesaka, "The evaluation of iron status in patients receiving recombinant human erythropoietin," Journal of the American Society of Nephrology, vol. 7, pp. 654-657, 1996.

[3] A. Mast, "The clinical utility of peripheral blood tests in the diagnosis of iron deficiency anemia," Bloodline, vol. 1, pp. 7-9, 2001.

[4] D. Coyne, "Iron indices: what do they really mean?" Kidney International, vol. 69, no. 101, supplement, pp. S4-S8, 2006.

[5] I. C. Macdougall, I. Cavill, B. Hulme, et al., "Detection of functional iron deficiency during erythropoietin treatment: a new approach," British Medical Journal, vol. 304, no. 6821, pp. 225-226, 1992.

[6] J. F. Navarro and M. L. Macia, "Hypochromic red cells as an indicator of iron deficiency," The Journal of Rheumatology, vol. 24, no. 4, pp. 804-805, 1997.

[7] S. Fishbane, C. Galgano, R. C. Langley Jr., W. Canfield, and J. K. Maesaka, "Reticulocyte hemoglobin content in the evaluation of iron status of hemodialysis patients," Kidney International, vol. 52, no. 1, pp. 217-222, 1997.

[8] A. E. Mast, M. A. Blinder, Q. Lu, S. Flax, and D. J. Dietzen, "Clinical utility of the reticulocyte hemoglobin content in the diagnosis of iron deficiency," Blood, vol. 99, no. 4, pp. 14891491, 2002.

[9] C. Brugnara, "Iron deficiency and erythropoiesis: new diagnostic approaches," Clinical Chemistry, vol. 49, no. 10, pp. 1573-1578, 2003.

[10] I. C. Macdougall, "Merits of percentage hypochromic red cells as a marker of functional iron deficiency," Nephrology Dialysis Transplantation, vol. 13, no. 4, pp. 847-849, 1998.

[11] C. Bovy, A. Gothot, J.-M. Krzesinski, and Y. Beguin, "Mature erythrocyte indices: new markers of iron availability," Haematologica, vol. 90, no. 4, pp. 549-551, 2005.

[12] I. C. Macdougall, W. H. Horl, C. Jacobs, et al., "European best practice guidelines 6-8: assessing and optimizing iron stores," Nephrology Dialysis Transplantation, vol. 15, no. 4, pp. 20-32, 2000 .
[13] S. Kotisaari, J. Romppanen, I. Penttila, and K. Punnonen, "The Advia 120 red blood cell and reticulocyte indices are useful in diagnosis of iron-deficiency anemia," European Journal of Haematology, vol. 68, no. 3, pp. 150-156, 2002.

[14] F. Locateli, P. Aljama, P. Barany, et al., "Revised European best practice guidelines for the management of anaemia in patients with chronic renal failure," Nephrology Dialysis Transplantation, vol. 19, supplement 2, pp. 1-47, 2004.

[15] M. Buttarello, V. Temporin, R. Ceravolo, G. Farina, and P. Burian, "The new reticulocyte parameter RET Y of the Sysmex $\mathrm{XE}$ 2100. Its use in the diagnosis and monitoring of post treatment sideropenic anemia," American Journal of Clinical Pathology, vol. 121, pp. 489-495, 2004.

[16] L. Thomas, S. Franck, M. Messinger, J. Linssen, M. Thome, and C. Thomas, "Reticulocyte hemoglobin measurementcomparison of two methods in the diagnosis of iron-restricted erythropoiesis," Clinical Chemistry and Laboratory Medicine, vol. 43, no. 11, pp. 1193-1202, 2005.

[17] A. E. Mast, M. A. Blinder, and D. J. Dietzen, "Reticulocyte hemoglobin content," American Journal of Hematology, vol. 83, no. 4, pp. 307-310, 2008.

[18] E. Urrechaga, L. Borque, and J. F. Escanero, "Potential utility of the new sysmex XE 5000 red blood cell extended parameters in the study of disorders of iron metabolism," Clinical Chemistry and Laboratory Medicine, vol. 47, no. 11, pp. 1411-1416, 2009.

[19] E. Urrechaga, "Clinical utility of the new beckman-coulter parameter red blood cell size factor in the study of erithropoiesis," International Journal of Laboratory Hematology, vol. 31, no. 6, pp. 623-629, 2009.

[20] E. Urrechaga, "The new mature red cell parameter, low haemoglobin density of the Beckman-Coulter LH750: clinical utility in the diagnosis of iron deficiency," International Journal of Laboratory Hematology, vol. 32, no. 1, part 1, pp. e144-e150, 2010.

[21] "NKF-K/DOQI clinical practice guidelines and clinical practice recommendations for anemia in chronic kidney disease in adults," American Journal of Kidney Diseases, vol. 47, no. 5, supplement 3, pp. S11-S145, 2006.

[22] S. Fishbane, W. Shapiro, P. Dutka, O. F. Valenzuela, and J. Faubert, "A randomized trial of iron deficiency testing strategies in hemodialysis patients," Kidney International, vol. 60, no. 6, pp. 2406-2411, 2001.

[23] A. Besarab, "Evaluating iron sufficiency: a clearer view," Kidney International, vol. 60, no. 6, pp. 2412-2414, 2001.

[24] C. Brugnara, "A hematologic "gold standard" for irondeficient states?" Clinical Chemistry, vol. 48, no. 7, pp. 981982, 2002.

[25] C. Brugnara, M. R. Laufer, A. J. Friedman, K. Bridges, and O. Platt, "Reticulocyte hemoglobin content (CHr): early indicator of iron deficiency and response to therapy," Blood, vol. 83, no. 10, pp. 3100-3101, 1994.

[26] F. Valderrabano, W. H. Horl, C. Jacobs, et al., "European best practice guidelines 1-4: evaluating anaemia and initiating treatment," Nephrology Dialysis Transplantation, vol. 15, no. 4, pp. 8-14, 2000.

[27] C. Brugnara, M. R. Laufer, A. J. Friedman, and O. Platt, "Reticulocyte hemoglobin content $(\mathrm{CHr})$ : early indicator of iron deficiency and response to therapy," Blood, vol. 82, A93, 1993.

[28] C. Thomas and L. Thomas, "Biochemical markers and hematologic indices in the diagnosis of functional iron deficiency," Clinical Chemistry, vol. 48, no. 7, pp. 1066-1076, 2002.

[29] U. Arndt, J. P. Kaltwasser, R. Gottschalk, D. Hoelzer, and B. Möller, "Correction of iron-deficient erythropoiesis in the 
treatment of anemia of chronic disease with recombinant human erythropoietin," Annals of Hematology, vol. 84, no. 3, pp. 159-166, 2005.

[30] C. Bovy, A. Gothot, P. Delanaye, X. Warling, J.-M. Krzesinski, and Y. Beguin, "Mature erythrocyte parameters as new markers of functional iron deficiency in haemodialysis: sensitivity and specificity," Nephrology Dialysis Transplantation, vol. 22, no. 4, pp. 1156-1162, 2007. 


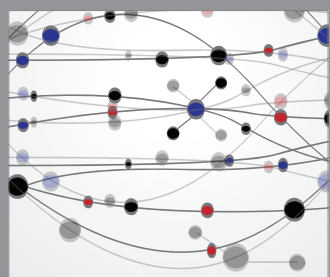

The Scientific World Journal
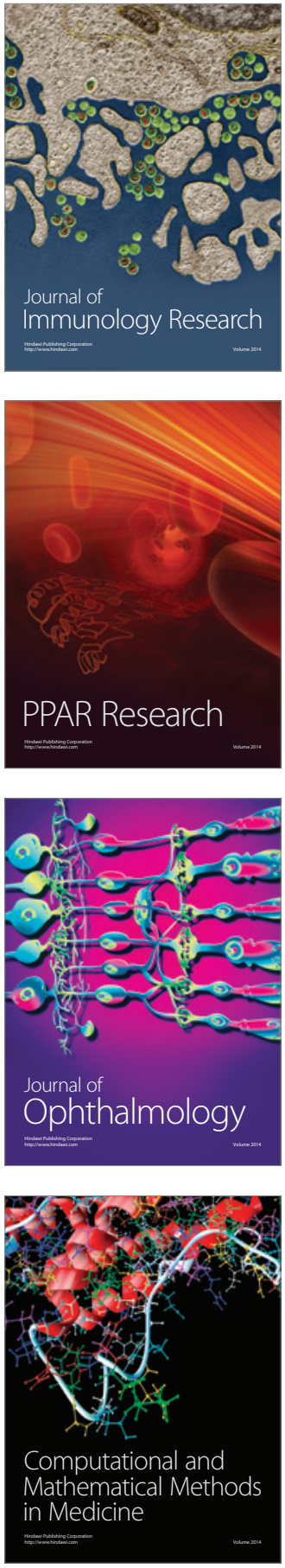

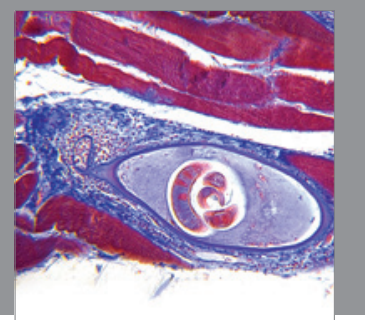

Gastroenterology

Research and Practice
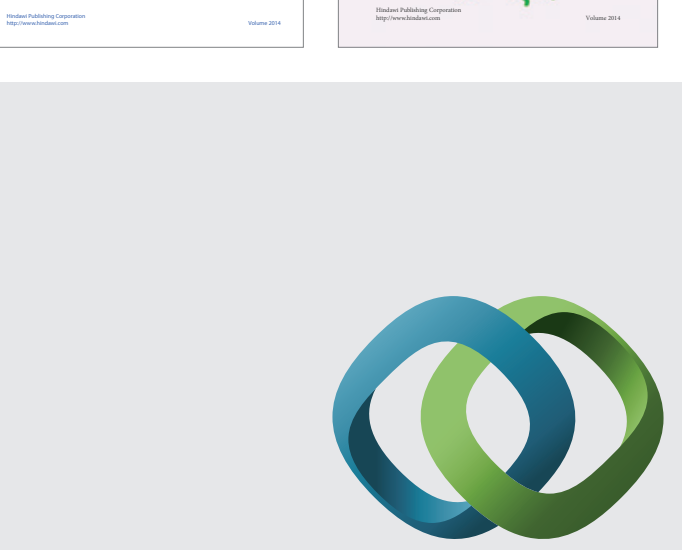

\section{Hindawi}

Submit your manuscripts at

http://www.hindawi.com
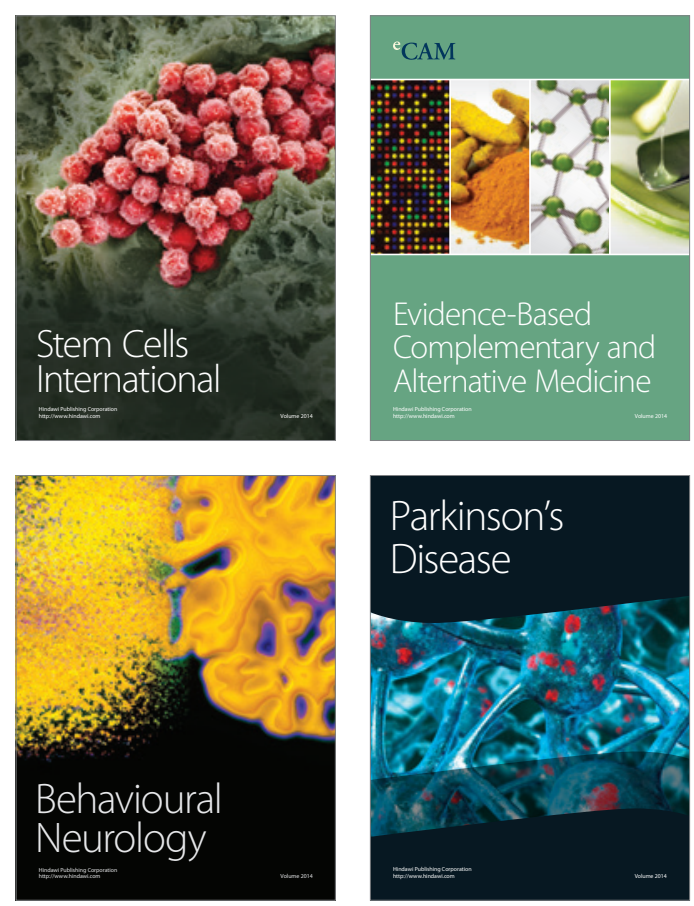

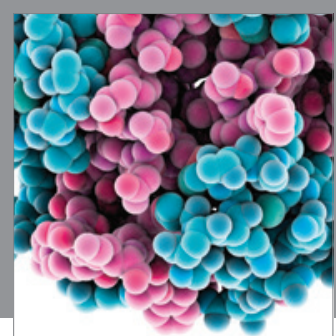

Journal of
Diabetes Research

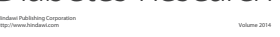

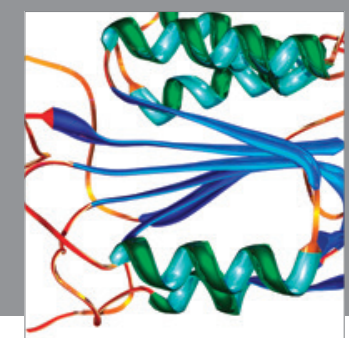

Disease Markers
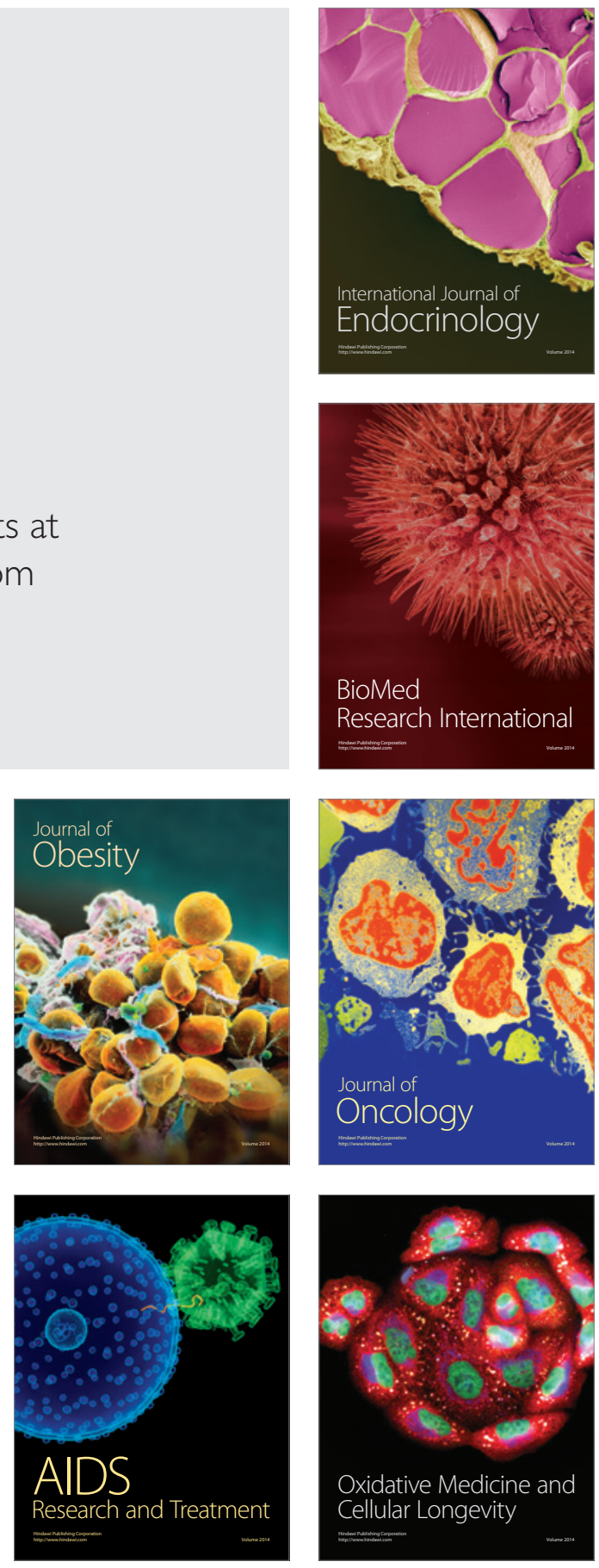